\title{
Canadian medical image app number one in the US
}

A smartphone app for sharing medical images - and commenting on them - was the number one free health care app in the United States within two weeks of its launch on May 28. The app, called Figure 1, allows doctors to share interesting images or photos of unique medical conditions, while building what could become a huge visual reference library.

"The grand vision is to have a free, open access medical repository that any health care professional can contribute to and can comment on," said Dr. Josh Landy, the Toronto, Ontariobased critical care physician who came up with the idea for the app which is available through iTunes. "Something like that doesn't exist and I think it would be a tremendous educational facility to have thousands of assets that have been collated and curated by the greater medical community."

He said most physicians use smart phones in their work and already photo-share, usually among colleagues they know, so the app seemed a natural progression.

Patient privacy is paramount: no faces, tattoos or other identifying information are permitted. These rules are detailed in the apps FAQ. And if a doctor accidentally uploads a face, the app has a face detection algorithm that blocks it.

The Figure 1 app comes with consent forms that patients can sign on their smartphone. And tools make it easy to anonymize an image. "If you take a picture of an X-ray and there's a name in the corner, just draw your fin-

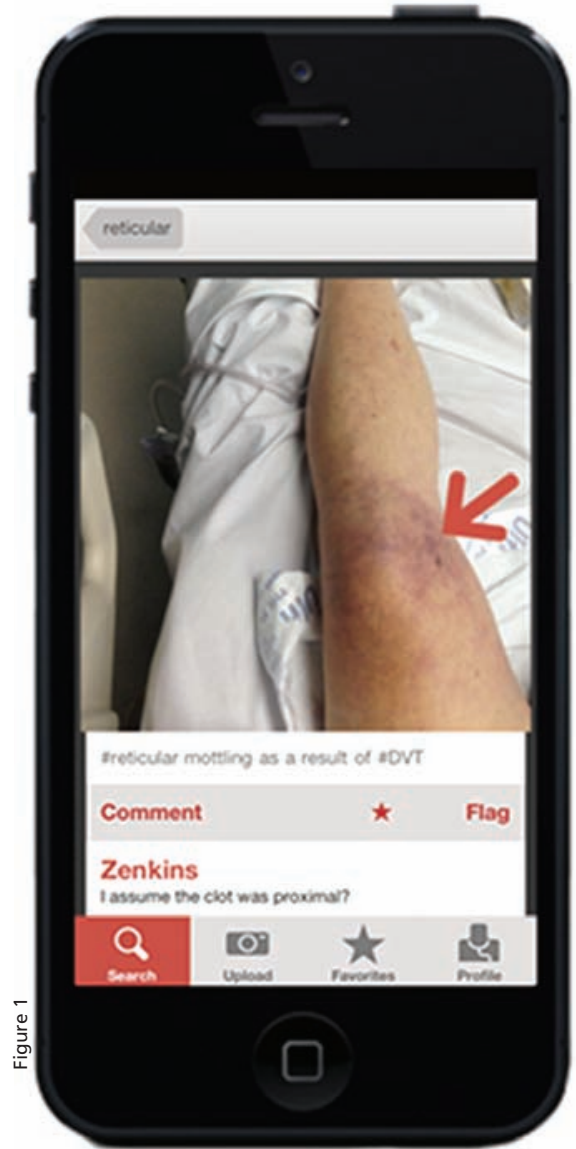

The Figure 1 app allows doctors to share interesting images or photos of unique medical conditions, while building what could become a huge visual reference library.

ger across it. You can also use one of the tools to annotate and we've also got an arrow you can use to indicate the site of interest," said Landy

Some users have described Figure 1 as an "Instagram for the medical community" but Landy who is pas- sionate about "medical education and technology" prefers to think of it as more of a Wikipedia model. "This is medical education. It's not diagnostic, it's educational."

That means the app is not designed as a utility, but as a way to share knowledge. "It works really well," said David Maslove, a critical care specialist in the Toronto area with an interest in biomedical informatics, who beta-tested Figure 1. "There's a general interest among physicians to share things, that's the nature of our training and the way we learn. Increasingly we share things online or though mobile devices especially when we see something unusual that would make a good exemplar.'

It took less than two months to build the app, but many more months of due diligence especially on the legal and privacy fronts.

For now, Figure 1 is only available in North America and only for iPhones. But the team is working on a web platform for desktops and also one for the Android platform. It's hoped that both will be available later this year.

Although the app is freely available to the public, around $90 \%$ of those downloading it are health care professionals according to Movable Science, the Toronto start-up company founded by Landy and two friends: an experienced $\mathrm{iOS}$ developer and a business expert. The app was launched in partnership with Ryerson University's digital media zone entrepreneurial arm. Karen Birchard, Charlottetown, PEI

CMAJ 2013. DOI:10.1503/cmaj.109-4527 